\title{
A test of homogeneity of Hardy-Weinberg disequilibrium across strata
}

\author{
Xiao-Lin Yin ${ }^{1}$, Wen-Qing Ma ${ }^{1}$, Man-Lai Tang ${ }^{2}$ and Jianhua Guo ${ }^{*, 1}$ \\ ${ }^{1}$ Key Laboratory for Applied Statistics of MOE, School of Mathematics and Statistics, Northeast Normal University, \\ ChangChun, China; ${ }^{2}$ Department of Mathematics, Hong Kong Baptist University, Hong Kong, China
}

For genotype data being sampled from several strata with different allele frequencies, it is necessary to verify the assumption of homogeneity of Hardy-Weinberg disequilibrium across strata before testing Hardy-Weinberg law across strata. In practice, disequilibrium can be measured via fixation coefficients (ie, ratios of genotypic frequencies) or disequilibrium coefficients (ie, differences of genotypic frequencies). Test for homogeneity of Hardy-Weinberg disequilibrium using data from several populations has been derived according to fixation coefficients. In this article, using the likelihood score theory extended to nuisance parameters, we derive a homogeneity score test for comparing disequilibrium coefficients across several independent strata. Simulation results demonstrate that the homogeneity score test performs satisfactorily in the sense that its empirical size seldom exceeds the pre-chosen nominal level by more than $10 \%$ even for small sample sizes. Corresponding power and sample size formulae are provided as well. We illustrate our test with a real glyoxalase genotype data set.

European Journal of Human Genetics (2006) 14, 1223-1230. doi:10.1038/sj.ejhg.5201689; published online 26 July 2006

Keywords: disequilibrium coefficient; Hardy-Weinberg law; homogeneity; score test

\section{Introduction}

The law of Hardy-Weinberg equilibrium (HWE) states that in a large random mating population that is not affected by the evolutionary processes of mutation, migration, or selection, both the allele frequencies and the genotype frequencies are constant from generation to generation. ${ }^{1,2}$ Furthermore, the genotype frequencies are related to the allele frequencies by the square expansion of those allele frequencies. In other words, the law of HWE states that under a restrictive set of assumptions, it is possible to calculate the expected frequencies of genotypes in a population if the frequency of the different alleles in a population is known. The original descriptions of HWE become an important landmark in the history of population genetics, ${ }^{3}$ and it is now a common

*Correspondence: Professor I Guo, School of Mathematics and Statistics, Northeast Normal University, 5268 Renmin Street, ChangChun 130024, Jilin Province, P.R. China.

Tel: + 86431 5098576; Fax: + 86431 5098237;

E-mail: jhguo@nenu.edu.cn

Received 13 January 2006; revised 29 May 2006; accepted 1 June 2006; published online 26 July 2006 practice to verify whether observed genotypes conform to Hardy-Weinberg expectations. ${ }^{4,5}$

In a diallelic locus with alleles $A_{1}$ and $A_{2}$ across $K$ strata, let the genotypic array of the $k$ th $(k=1, \ldots, K)$ stratum be

$$
p_{11 k} A_{1} A_{1}+p_{12 k} A_{1} A_{2}+p_{22 k} A_{2} A_{2}
$$

Let $p_{k}$ be the allelic frequency of $A_{1}$ in the $k$ th stratum and $q_{k}=1-p_{k}(k=1, \ldots, K)$. Populations with genotypic frequencies satisfying $p_{11 k}=p_{k}^{2}, p_{12 k}=2 p_{k} q_{k}$, and $p_{22 k}=q_{k}^{2}(k=1, \ldots, K)$ are said to be in HWE at the locus under consideration. In studies of HWE, there are two widely used coefficients, namely the fixation and disequilibrium coefficients. ${ }^{6}$ For stratum $k(k=1, \ldots, K)$, the fixation and disequilibrium coefficients are defined by $f_{k}=1-p_{12 k} /\left(2 \sqrt{p_{11 k} p_{22 k}}\right)$ and $D_{k}=p_{k} q_{k}-p_{12 k} / 2$, respectively. Hence, the problem of testing HWE when individuals are sampled from several strata is equivalent to testing one of the following hypotheses:

$$
\begin{array}{ll}
\mathrm{H}_{0}^{\prime}: \theta_{k}=0 & \text { for all } k=1, \ldots, K \quad \text { versus } \\
\mathrm{H}_{1}^{\prime}: \theta_{k} \neq 0 & \text { for some } k,
\end{array}
$$

where $\theta_{k}=f_{k}$ or $D_{k}$. For statistical tests based on disequilibrium coefficient, one can refer to the work of Haldane ${ }^{7}$ and Smith. ${ }^{8}$ 
For test procedures based on functions of fixation coefficients (eg, $\left(1-f_{k}\right)^{2}$ ), one can consult the work of Emigh, ${ }^{9}$ Troendle and $\mathrm{Yu},{ }^{10}$ and Nam. ${ }^{11}$

It is noteworthy that any statistical procedure for testing the null hypothesis in (1) assumes that the measure of disequilibrium (ie, $\theta_{k}$ ) is constant across the strata. In this regard, it is important that one should consider testing the assumption of homogeneity of the measure of disequilibrium across strata before any testing of the null hypothesis in (1). For this purpose, we consider the following hypotheses:

$$
\mathrm{H}_{0}: \theta_{1}=\ldots=\theta_{k} \quad \text { versus } \mathrm{H}_{1}: \text { Not all } \theta_{k}^{\prime} \text { s are equal }
$$

Olson and Foley ${ }^{12}$ proposed a large-sample test and an exact test for verifying the null hypothesis $\mathrm{H}_{0}$ via a function of fixation coefficients, $\left(1-f_{k}\right)^{2}$. They also approximate the $P$-value of the exact test using a Markov chain Monte Carlo approach. Although the use of fixation coefficients to describe departures from HWE has some merit, it has the disadvantage that these parameters are estimated as ratios of genotypic frequencies. It is difficult to study sampling properties of ratio statistics. ${ }^{4,6}$ Besides, functions of fixation coefficients such as $\left(1-f_{k}\right)^{2}$ may possess infinite upper bound. On the other hand, there are advantages in working with a composite kind of quantity such as the disequilibrium coefficient. This is simply the difference between a frequency and its values expected when there are no association between alleles. Moreover, it is easy to show that disequilibrium coefficient $D_{k}$ satisfies $\max \left\{-p_{k}^{2}\right.$, $\left.-q_{k}^{2}\right\} \leqslant D_{k} \leqslant p_{k} q_{k}$. Unfortunately, test of homogeneity of disequilibrium coefficients across several strata has not been considered in the literature yet. Therefore, the objective of this study is to develop a new homogeneity score statistic for testing the null hypothesis in (2) based on disequilibrium coefficients. We first develop the theory and method and then demonstrate the advantage of our method over the method proposed by Olson and Foley ${ }^{12}$ via Monte Carlo simulation studies. We also derive the approximate power and sample size formulae, which are necessary in design of studies. Finally, we illustrate our test with a real glyoxalase genotype data set.

\section{Method}

\section{Homogeneity test}

Let $X_{i j k}(i \leqslant j=1,2$ and $k=1, \ldots, K)$ be the number of individuals with genotype $A_{i} A_{j}$ in the $k$ th population with $n_{k}=X_{11 k}+X_{12 k}+X_{22 k}$. Let $M\left(n_{k},\left\{p_{i j k}\right\}\right)$ denote the trinomial distribution with parameter vector $\left(p_{11 k}, p_{12 k}, p_{22 k}\right)$. Hence, we have $\left\{X_{i j k}: i, j=1,2 ; i \leqslant j\right\} \sim M\left(n_{k},\left\{p_{i j k}\right\}\right)$ for $k=1, \ldots, K$. In this article, we are interested to test the homogeneity hypothesis in (2) with $\theta_{k}=D_{k}$. That is,

$$
\mathrm{H}_{0}: D_{1}=\cdots=D_{k} \text { versus } H_{1}: \text { Not all } D_{k}^{\prime} \text { s are equal, }
$$

where $D_{k}=p_{k} q_{k}-p_{12 k} / 2$. All subsequent results are obtained under the assumptions that $K$ is fixed and $n_{k}$ is sufficiently large for $k=1,2, \ldots, K$.
Note that $p_{11 k}=p_{k}^{2}+D_{k}, \quad p_{12 k}=2\left(p_{k} q_{k}-D_{k}\right) \quad$ and $p_{22 k}=q_{k}^{2}+D_{k}$, the log-likelihood for the $k$ th strata can be expressed in terms of $D_{k}$ and $p_{k}(k=1, \ldots, K)$ as

$$
\begin{aligned}
l_{k}\left(D_{k}, p_{k}\right)= & x_{11 k} \ln \left(p_{k}^{2}+D_{k}\right)+x_{12 k} \ln \left(2\left(p_{k} q_{k}-D_{k}\right)\right) \\
& +x_{22 k} \ln \left(q_{k}^{2}+D_{k}\right)
\end{aligned}
$$

Let $D$ denote the common disequilibrium coefficient under $\mathrm{H}_{0}$ and $\mathbf{p}=\left(p_{1}, \ldots, p_{K}\right)^{\prime}$ the nuisance parameter vector. Under $\mathrm{H}_{0}$, the total log-likelihood for all $K$ strata is given by

$$
l(D, \mathbf{p})=\sum_{k=1}^{K} l_{k}\left(D, p_{k}\right)
$$

Hence, the efficient scores for the $k$ th stratum (ie, the firstorder derivatives of $l_{k}\left(D, p_{k}\right)$ with respect to $D$ and $\left.p_{k}\right)$ are given by

$$
\begin{gathered}
H_{k D}\left(D, p_{k}\right)=\frac{\partial l_{k}\left(D, p_{k}\right)}{\partial D}=\frac{x_{11 k}}{p_{k}^{2}+D}-\frac{x_{12 k}}{p_{k} q_{k}-D}+\frac{x_{22 k}}{q_{k}^{2}+D}, \\
H_{k} p_{k}\left(D, p_{k}\right)=\frac{\partial l_{k}\left(D, p_{k}\right)}{\partial p_{k}}=\frac{2 x_{11 k} p_{k}}{p_{k}^{2}+D}+\frac{x_{12 k}\left(1-2 p_{k}\right)}{p_{k} q_{k}-D}-\frac{2 x_{22 k} q_{k}}{q_{k}^{2}+D}
\end{gathered}
$$

Let $\hat{D}$ and $\hat{\mathrm{p}}$ be the maximum-likelihood estimates (MLEs) of $D$ and $\mathrm{p}$ under the null hypothesis $\mathrm{H}_{0}$. In this case, $\hat{D}$ and $\hat{\mathrm{p}}$ must satisfy the following $K+1$ equations:

$$
\begin{aligned}
& \sum_{k=1}^{K} H_{k D}\left(\hat{D}, \hat{p}_{k}\right)=0, \\
& \text { and } \\
& H_{k} p_{k}\left(\hat{D}, \hat{p}_{k}\right)=0, \quad k=1,2, \ldots, K
\end{aligned}
$$

Denote

$$
\begin{aligned}
V_{k D D}\left(D, p_{k}\right)= & \frac{\partial H_{k D}\left(D, p_{k}\right)}{\partial D}=-\frac{x_{11 k}}{\left(p_{k}^{2}+D\right)^{2}} \\
& -\frac{x_{12 k}}{\left(p_{k} q_{k}-D\right)^{2}}-\frac{x_{22 k}}{\left(q_{k}^{2}+D\right)^{2}}, \\
V_{k D} p_{k}\left(D, p_{k}\right)= & \frac{\partial H_{k D}\left(D, p_{k}\right)}{\partial p_{k}}=-\frac{2 x_{11 k} p_{k}}{\left(p_{k}^{2}+D\right)^{2}} \\
& +\frac{x_{12 k}\left(1-2 p_{k}\right)}{\left(p_{k} q_{k}-D\right)^{2}}+\frac{2 x_{22 k} q_{k}}{\left(q_{k}^{2}+D\right)^{2}},
\end{aligned}
$$

and

$$
\begin{aligned}
V_{k} p_{k} p_{k}\left(D, p_{k}\right)= & \frac{\partial H_{k} p_{k}\left(D, p_{k}\right)}{\partial p_{k}}=\frac{2 x_{11 k}\left(D-p_{k}^{2}\right)}{\left(p_{k}^{2}+D\right)^{2}} \\
& +\frac{x_{12 k}\left(2 D-p_{k}^{2}-q_{k}^{2}\right)}{\left(p_{k} q_{k}-D\right)^{2}}+\frac{2 x_{22 k}\left(D-q_{k}^{2}\right)}{\left(q_{k}^{2}+D\right)^{2}}
\end{aligned}
$$

In addition, denote $I_{k D} \mid p_{k}=I_{k D D}-I_{k D p_{k}}^{2} / I_{k p_{k} p_{k}}$, where

$$
\begin{aligned}
& I_{k D D}=-E\left(V_{k D D}\right)=\frac{n_{k}\left(p_{k} q_{k}+D\right)}{\left(p_{k}^{2}+D\right)\left(p_{k} q_{k}-D\right)\left(q_{k}^{2}+D\right)}, \\
& I_{k D} p_{k}=-E\left({ }_{V k D} p_{k}\right)=\frac{2 n_{k}\left(2 p_{k}-1\right) D}{\left(p_{k}^{2}+D\right)\left(p_{k} q_{k}-D\right)\left(q_{k}^{2}+D\right)},
\end{aligned}
$$


and

$$
\begin{aligned}
& I_{k} P_{k} P_{k}=-E\left(V_{k} P_{k} P_{k}\right) \\
& =2 n_{k} \frac{\left(p_{k}^{2}+D\right)\left(q_{k}^{2}+D\right)^{2}+\left(p_{k} q_{k}-D\right)^{3}+\left(p_{k}^{2}+D\right)^{2}\left(q_{k}^{2}+D\right)-4 D^{2} .}{\left(p_{k}^{2}+D\right)\left(p_{k} q_{k}-D\right)\left(q_{k}^{2}+D\right)} .
\end{aligned}
$$

Hence, the likelihood score test for testing $\mathrm{H}_{0}$ : $D_{1}=\cdots=D_{K}$ is given by

$$
X^{2}=\sum_{k=1}^{K} \frac{H_{k D}^{2}\left(\hat{D}, \hat{p}_{k}\right)}{I_{k D} \mid P_{k}\left(\hat{D}, \hat{p}_{k}\right)},
$$

which is asymptotically distributed as a $\chi^{2}$ variate with $K-1$ degrees of freedom under $\mathrm{H}_{0}$. Unfortunately, we note that $\hat{D}$ and $\hat{p}$ cannot be expressed in closed form and this makes the likelihood score test $X^{2}$ less appealing in real applications. To over this issue, using the theory of homogeneity score test extended to nuisance parameters, ${ }^{13}$ we consider the following modified score statistic:

$$
X^{2 *}=\sum_{k=1}^{K} \frac{H_{k D}^{2}\left(D^{*}, p_{k}^{*}\right)}{I_{k D \mid p_{k}}\left(D^{*}, p_{k}^{*}\right)}-\frac{\left[\sum_{k=1}^{K} H_{k D}^{2}\left(D^{*}, p_{k}^{*}\right)\right]^{2}}{\sum_{k=1}^{K} I_{k D \mid p_{k}}\left(D^{*}, p_{k}^{*}\right)},
$$

where $D^{*}$ and $\mathbf{p}^{*}$ are any consistent estimators of $D$ and p, respectively. To this end, we choose $D^{*}$ to be $\sum_{k=1}^{k}$ $\left(4 x_{11 k} x_{22 k} / x_{12 k}^{2}-1\right) / \sum_{k=1}^{k}\left(4 n_{k}^{2} / x_{12 k}^{2}\right)$ and $p^{*}{ }_{k}$ be the solution to the following equation:

$$
H_{k p_{k}}\left(D^{*}, p_{k}\right) \equiv \frac{2 x_{11 k} p_{k}}{p_{k}^{2}+D^{*}}+\frac{x_{12 k}\left(1-2 p_{k}\right)}{p_{k} q_{k}-D^{*}}-\frac{2 x_{22 k} q_{k}}{q_{k}^{2}+D^{*}}=0,
$$

or equivalently the following quintic polynomial equation,

$$
a_{0}+a_{1} p_{k}+a_{2} p_{k}^{2}+a_{3} p_{k}^{3}+a_{4} p_{k}^{4}+a_{5} p_{k}^{5}=0,
$$

where $\quad a_{0}=x_{12 k} D^{*}\left(1+D^{*}\right)+2 x_{22 k}\left(D^{*}\right)^{2}, \quad a_{1}=-2\left(n_{k} D^{*}\right.$ $\left.\left(1+D^{\star}\right)+x_{12 k} D^{*}\right), a_{2}=6 n_{k} D^{*}+2 x_{11 k}+x_{12 k}, a_{3}=-2\left(2 n_{k} D^{*}+\right.$ $\left.n_{k}+2 x_{11 k}+x_{12 k}\right), \quad a_{4}=4 n_{k}+2 x_{11 k}+x_{12 k}, \quad$ and $a_{5}=-2 n_{k}$. Here, $D^{*}$ is analogous to the Mantel-Haenszel estimator ${ }^{14}$ and is a consistent estimator to $D$. However, it is not an efficient estimator to $D$ in general. The proof of consistency and the condition to attain asymptotic efficiency for $D^{*}$ is given in Appendix A. We note that the calculation of $I_{k D \mid p_{k}}$ in (3) could be tedious. Nonetheless, it is easy to show that $I_{k D \mid p_{k}}$ is simply given by $n_{k} / w_{k}\left(D, p_{k}\right)$ with $w_{k}\left(D, p_{k}\right)=\left(p_{k}^{2}+D\right)$ $\left(q_{k}^{2}+D\right)^{2}+2\left(p_{k} q_{k}-D\right)^{3}+\left(p_{k}^{2}+D\right)^{2}\left(q_{k}^{2}+D\right)-4 D^{2}$ (see Appendix B for the proof). Similarly, $X^{2 *}$ is asymptotically distributed as a $\chi^{2}$ variate with $K-1$ degrees of freedom under $\mathrm{H}_{0}$. Therefore, the homogeneity hypothesis $\mathrm{H}_{0}$ is rejected at level $\alpha$ if $X^{2 *} \geqslant \chi_{K-1,(1-\alpha)}^{2}$, where $\chi_{K-1,(1-\alpha)}^{2}$, is the $100 \times(1-\alpha)$ percentile point of the $\chi^{2}$ distribution with $K-1$ degrees of freedom. Finally, it is noteworthy that if the consistent estimators of $D$ and $\mathbf{p}$ are the constrained maximumlikelihood estimators under $\mathrm{H}_{0}$, then the second term of (3) vanishes, since $\sum_{k=1}^{K} H_{k D}\left(D^{*}, p_{k}^{*}\right)=0$, and (3) reduces to the likelihood score statistic.
Asymptotic power and sample size formulae

In this section, we aim to derive the asymptotic power and sample size formulae ${ }^{15}$ based on $X^{2 *}$. For these purposes, we assume $n_{k}=n b_{k}$ for some $n$ and $b_{k}>0$. Let $\overline{D_{k}}$ and $\overline{p_{k}}$ be the true parameter values for $D_{k}$ and $p_{k}$ under the alternative $H_{1}$, where $k=1,2, \ldots, K$ and $\bar{D}_{k} \neq \bar{D}_{j}$ for some $k \neq j$. Hence, the asymptotic power of the homogeneity score test $X^{2 \star}$ at $\alpha$ level is given by

$$
\operatorname{Pr}\left(X^{2 *}>\chi_{K-1,(1-\alpha)}^{2} \mid H_{1}\right)=\operatorname{Pr}\left(\chi_{K-1}^{2}(\delta) \geqslant \chi_{K-1,(1-\alpha)}^{2}\right),
$$

where $\chi_{K-1}^{2}(\delta)$ denotes the non-central $\chi^{2}$ distribution with $K-1$ degrees of freedom and the non-centrality parameter $\delta$ is equal to

$$
\begin{aligned}
\delta= & n\left\{\sum_{k=1}^{K} \frac{\left[b_{k}\left(\frac{\bar{p}_{k}^{2}+\bar{D}_{k}}{p_{k}^{2}+D}-\frac{2\left(\bar{p}_{k} \bar{q}_{k}-D_{k}\right)}{p_{k} q_{k}-D}+\frac{\bar{q}_{k}^{2}+\bar{D}_{k}}{q_{k}^{2}+D}\right)\right]^{2}}{b_{k} / w_{k}\left(D, p_{k}\right)}\right. \\
& \left.-\frac{\left[\sum_{k=1}^{K} b_{k}\left(\frac{\bar{p}_{k}^{2}+\bar{D}_{k}}{p_{k}^{2}+D}-\frac{2\left(\bar{p}_{k} \bar{q}_{k}-\bar{D}_{k}\right)}{p_{k} q_{k}-D}+\frac{\bar{q}_{k}^{2}+\bar{D}_{k}}{q_{k}^{2}+D}\right)\right]^{2}}{\sum_{k=1}^{K}\left[b_{k} / w_{k}\left(D, p_{k}\right)\right]}\right\},
\end{aligned}
$$

with $\overline{q_{k}}=1-\overline{p_{k}}$

$$
D=\sum_{k=1}^{K}\left(\frac{\left(\bar{p}_{k}^{2}+\bar{D}\right)\left(\bar{q}_{k}^{2}+\bar{D}\right)}{\left(\bar{p}_{k} \bar{q}_{k}-\bar{D}_{k}\right)^{2}}-1\right) / \sum_{k=1}^{K}\left[1 /\left(\bar{p}_{k} \bar{q}_{k}-\bar{D}_{k}\right)^{2}\right] \text {, }
$$

and $p_{k}$ is the solution to the following equation:

$$
\bar{a}_{0}+\bar{a}_{1} p_{k}+\bar{a}_{2} p_{k}^{2}+\bar{a}_{3} p_{k}^{3}+\bar{a}_{4} p_{k}^{4}+\bar{a}_{5} p_{k}^{5}=0,
$$

where $\quad \overline{a_{0}}=2\left(\overline{p_{k}} \bar{q}_{k}-\overline{D_{k}}\right) D(1+D)+2\left(\bar{q}_{k}^{2}+\overline{D_{k}}\right) D^{2}, \quad \overline{a_{1}}=-2 D$ $(1+D)+4\left(\overline{p_{k}} \overline{q_{k}}-\overline{D_{k}}\right) D, \quad \overline{a_{2}}=6 D+2 \overline{p_{k}}, \quad \overline{a_{3}}=-2\left(2 D+1+\overline{p_{k}}\right)$, $\overline{a_{4}}=4+\overline{p_{k}}$, and $\overline{a_{5}}=-2$.

As a result, the desirable sample size $n$ required to attain the power at $1-\beta$ with $\overline{D_{k}}$ and $\overline{p_{k}}$ being the true parameter values for $D_{k}$ and $p_{k}$ under the alternative $H_{1}$ at nominal level $\alpha$ can be determined from the following equality:

$$
\chi_{K-1, \beta}^{2}(\delta)=\chi_{K-1,(1-\alpha)}^{2},
$$

where $\chi_{K-1, \beta}^{2}(\delta)$ is the $100 \times \beta$ percentage point of the non-central $\chi^{2}$ distribution with $K-1$ degrees of freedom with non-centrality parameter being $\delta$. The value of $n$ can be readily obtained by solving the equation given in (4).

\section{Simulation}

We evaluate the performance of our proposed homogeneity score test in terms of type I error rate and power. For type I error rate, we include the homogeneity test proposed by Olson and Foley ${ }^{12}$ in our comparison study. In their case, they adopted a function of fixation coefficients as the measure for Hardy-Weinberg disequilibrium. Specifically, they were interested to test the homogeneity hypothesis in (2) with $\theta_{k}=\left(1-f_{k}\right)^{2}$. That is,

$\mathrm{H}_{0}^{*}:\left(1-f_{1}\right)^{2}=\cdots=\left(1-f_{K}\right)^{2}$ versus $\mathrm{H}_{1}^{*}$ : Not all $\left(1-f_{k}\right)^{2}$ are equal, and their proposed statistic for testing the above 
hypotheses is given by

$$
T_{\text {homog }}^{2}=\sum_{k=1}^{k} \frac{h_{k}^{2}(\hat{\theta})}{\hat{V} a r\left[h_{k}(\hat{\theta})\right]},
$$

where $\theta=\left(\sum_{k=1}^{K}\left(\left(x_{12 \mathrm{k}}^{2}-x_{12 k}\right) /\left(2\left(2 n_{k}-1\right)\right)\right) /\left(\sum_{k=1}^{K}\left(\left(2 x_{11 \mathrm{k}}^{2}-\right.\right.\right.\right.$ $\left.\left.\left.x_{22 k}\right) /\left(2 n_{k}-1\right)\right)\right), \quad h_{k}(\theta)=x_{12 \mathrm{k}}^{2}-x_{12 k}-4 \theta x_{11 k} x_{22 k}, \quad$ and Vâr $\left[h_{k}(\theta)\right]=4\left(x_{11 k}^{3}-3 x_{11 k}^{2}+2 x_{11 k}\right)(1-\theta)+2\left(x_{11 k}^{2}-x_{11 k}\right)\left(2 n_{k} \theta-3 \theta\right.$ $+2)$ for $k=1, \ldots, K$. We would like to point out here that our proposed homogeneity score test (ie, $X^{2 *}$ ) and Olson and Foley's test (ie, $T_{\text {homog }}^{2}$ ) can be fairly comparable only when $\theta_{k}=0$ for $k=1, \ldots, K$ in the null hypotheses $\mathrm{H}_{0}$ and $\mathrm{H}_{0}{ }^{*}$ (ie, $\mathrm{H}^{\prime}{ }_{0}$ in (1)). In the present comparisons, we consider both the asymptotic (denoted as $T_{\text {homog,a }}^{2}$ ) and exact (denoted as $T_{\text {homog,e }}$ ) versions of $T_{\text {homog. }}^{2}$ For the implementation of $T_{\text {homog,e }}^{2}$ one can refer to Olson and Foley (1996, p 975). Here, we investigate type I error rates of $X^{2 \star}$ and $T_{\text {homog,a }}$ for small (eg, $n_{k}=20$ and 30) to large sample sizes (eg, $n_{k}=50-200$ ) when $\theta_{k}=0$ for $k=1, \ldots, K$. As $T_{h o m o g, e}^{2}$ is computationally intensive for large sample sizes, we consider its small-sample behavior only. Results of Monte Carlo experiments with 5000 repetitions for different designed allele probabilities $p^{\prime}{ }_{k} s$ with $k=1, \ldots, K$ and $K=3$ and 5 at 0.05 nominal level are summarized in Tables 1 (for small sample sizes) and 2 (for moderate to large sample sizes).

As expected, the exact test $T_{\text {homog,e }}^{2}$ is always conservative (ie, its type I error rates are always less than the preassigned nominal level). The empirical type I error rates of our asymptotic homogeneity score test $X^{2 *}$ are satisfactorily close to the nominal 0.05 level for allelic probabilities being bounded away from 0 and 1, whereas those of the $T_{\text {homog, } a}^{2}$ are generally liberal (eg, more than 11 times of the given nominal level) even for large sample sizes. It is noteworthy that $X^{2 *}$ appears to be conservative than

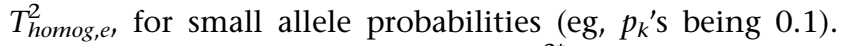
However, the conservativeness of $X^{2^{*}}$ vanishes with an increase in sample sizes and the computation of $X^{2^{*}}$ is much more simpler than $T_{\text {homog,e }}^{2}$

In view of the above observations, we prefer the proposed homogeneity score test $X^{2^{*}}$ (based on disequilibrium coefficients) to the existing homogeneity tests based on function of fixation coefficients (ie, $T_{\text {homog, } a}^{2}$ and $T_{\text {homog,e }}^{2}$ ). Hence, we exclude $T_{\text {homog,a }}^{2}$ and $T_{\text {homog,e }}^{2}$ in all subsequent evaluation and discussion. Table 3 further summarizes the type I error rate of $X^{2 *}$ for some non-zero (common) disequilibrium coefficients (ie, $D \neq 0$ ) under different settings. Again, the propose homogeneity score test performs satisfactorily in the sense that its empirical type I error rates are close to the pre-chosen nominal level and seldom exceed the nominal level by more than $10 \%$.

For power performance, the parameters and sample size are quite similar to those adopted in Table 3, except that $\left\{D_{k}\right\}$ are now specifically designed under $\mathrm{H}_{1}$. For this purpose, we set $D_{k}=D_{0}+\Delta(k-1)$. For $K=3$, we consider: (i) $D_{0}=-0.03, \Delta=0.03$ and (ii) $D_{0}=-0.05, \Delta=0.05$. For $K=5$, we consider: (i) $D_{0}=-0.06, \Delta=0.03$ and (ii) $D_{0}=-0.1, \Delta=0.05$. The results are reported in Table 4 . From the simulation results, the power of $X^{2^{*}}$ increases with the sample size $n$ or $\Delta$. For those settings with the same $\left\{D_{k}\right\}$, the one with varied allele probabilities across

Table 1 Empirical type I error rates for $X^{2^{*}}, T_{\text {homog, } a}^{2}$ and $T_{\text {homog, },}^{2}$ under $\mathrm{H}_{0}^{\prime}$ when $K=3$ and $K=5$

\begin{tabular}{|c|c|c|c|c|}
\hline$n$ & $p$ & $x^{2^{*}}$ & $T_{\text {homog, } a}^{2}$ & $T_{\text {homog, }}^{2}$ \\
\hline \multirow[t]{6}{*}{$20,20,20$} & $0.5,0.5,0.5$ & 0.058 & 0.133 & 0.041 \\
\hline & $0.5,0.4,0.3$ & 0.047 & 0.141 & 0.041 \\
\hline & $0.5,0.3,0.1$ & 0.046 & 0.215 & 0.033 \\
\hline & $0.3,0.3,0.3$ & 0.029 & 0.144 & 0.035 \\
\hline & $0.3,0.2,0.1$ & 0.023 & 0.228 & 0.024 \\
\hline & $0.1,0.1,0.1$ & 0.006 & 0.420 & 0.010 \\
\hline \multirow[t]{6}{*}{$30,30,30$} & $0.5,0.5,0.5$ & 0.055 & 0.104 & 0.046 \\
\hline & $0.5,0.4,0.3$ & 0.047 & 0.113 & 0.046 \\
\hline & $0.5,0.3,0.1$ & 0.045 & 0.144 & 0.041 \\
\hline & $0.3,0.3,0.3$ & 0.033 & 0.107 & 0.041 \\
\hline & $0.3,0.2,0.1$ & 0.026 & 0.141 & 0.027 \\
\hline & $0.1,0.1,0.1$ & 0.004 & 0.229 & 0.011 \\
\hline \multirow[t]{6}{*}{$20,20,20,20,20$} & $0.5,0.5,0.5,0.5,0.5$ & 0.059 & 0.192 & 0.048 \\
\hline & $0.5,0.4,0.3,0.2,0.1$ & 0.041 & 0.294 & 0.049 \\
\hline & $0.5,0.3,0.1,0.3,0.5$ & 0.047 & 0.280 & 0.047 \\
\hline & $0.3,0.3,0.3,0.3,0.3$ & 0.021 & 0.223 & 0.047 \\
\hline & $0.1,0.3,0.5,0.3,0.1$ & 0.032 & 0.363 & 0.048 \\
\hline & $0.1,0.1,0.1,0.1,0.1$ & 0.008 & 0.581 & 0.022 \\
\hline \multirow[t]{6}{*}{$30,30,30,30,30$} & $0.5,0.5,0.5,0.5,0.5$ & 0.054 & 0.151 & 0.047 \\
\hline & $0.5,0.4,0.3,0.2,0.1$ & 0.040 & 0.190 & 0.046 \\
\hline & $0.5,0.3,0.1,0.3,0.5$ & 0.046 & 0.178 & 0.048 \\
\hline & $0.3,0.3,0.3,0.3,0.3$ & 0.028 & 0.156 & 0.047 \\
\hline & $0.1,0.3,0.5,0.3,0.1$ & 0.033 & 0.221 & 0.049 \\
\hline & $0.1,0.1,0.1,0.1,0.1$ & 0.006 & 0.330 & 0.026 \\
\hline
\end{tabular}


Table 2 Empirical type I error rates for $X^{2^{*}}$ and $T_{\text {homog, } a}^{2}$ under $\mathrm{H}^{\prime}{ }_{0}$ when $K=3$ and $K=5$

\begin{tabular}{|c|c|c|c|}
\hline$n$ & $P$ & $x^{2^{*}}$ & $T_{\text {homog, }}^{2} a$ \\
\hline \multirow[t]{6}{*}{$50,50,50$} & $0.5,0.5,0.5$ & 0.053 & 0.083 \\
\hline & $0.5,0.4,0.3$ & 0.046 & 0.083 \\
\hline & $0.5,0.3,0.1$ & 0.043 & 0.105 \\
\hline & $0.3,0.3,0.3$ & 0.041 & 0.086 \\
\hline & $0.3,0.2,0.1$ & 0.034 & 0.098 \\
\hline & $0.1,0.1,0.1$ & 0.003 & 0.124 \\
\hline \multirow[t]{6}{*}{$100,100,100$} & $0.5,0.5,0.5$ & 0.051 & 0.065 \\
\hline & $0.5,0.4,0.3$ & 0.051 & 0.070 \\
\hline & $0.5,0.3,0.1$ & 0.046 & 0.075 \\
\hline & $0.3,0.3,0.3$ & 0.048 & 0.070 \\
\hline & $0.3,0.2,0.1$ & 0.036 & 0.075 \\
\hline & $0.1,0.1,0.1$ & 0.009 & 0.073 \\
\hline \multirow[t]{6}{*}{$200,200,200$} & $0.5,0.5,0.5$ & 0.050 & 0.058 \\
\hline & $0.5,0.4,0.3$ & 0.051 & 0.060 \\
\hline & $0.5,0.3,0.1$ & 0.047 & 0.061 \\
\hline & $0.3,0.3,0.3$ & 0.050 & 0.061 \\
\hline & $0.3,0.2,0.1$ & 0.043 & 0.064 \\
\hline & $0.1,0.1,0.1$ & 0.023 & 0.063 \\
\hline \multirow[t]{6}{*}{$50,100,200$} & $0.5,0.5,0.5$ & 0.050 & 0.072 \\
\hline & $0.5,0.4,0.3$ & 0.050 & 0.070 \\
\hline & $0.5,0.3,0.1$ & 0.046 & 0.068 \\
\hline & $0.3,0.3,0.3$ & 0.045 & 0.074 \\
\hline & $0.3,0.2,0.1$ & 0.042 & 0.071 \\
\hline & $0.1,0.1,0.1$ & 0.010 & 0.097 \\
\hline \multirow{6}{*}{ 100, 100, 100, 100, 100} & $0.5,0.5,0.5,0.5,0.5$ & 0.050 & 0.079 \\
\hline & $0.5,0.4,0.3,0.2,0.1$ & 0.042 & 0.096 \\
\hline & $0.5,0.3,0.1,0.3,0.5$ & 0.045 & 0.091 \\
\hline & $0.3,0.3,0.3,0.3,0.3$ & 0.046 & 0.086 \\
\hline & $0.1,0.3,0.5,0.3,0.1$ & 0.036 & 0.102 \\
\hline & $0.1,0.1,0.1,0.1,0.1$ & 0.008 & 0.106 \\
\hline \multirow[t]{6}{*}{$50,75,100,125,150$} & $0.5,0.5,0.5,0.5,0.5$ & 0.048 & 0.082 \\
\hline & $0.5,0.4,0.3,0.2,0.1$ & 0.043 & 0.094 \\
\hline & $0.5,0.3,0.1,0.3,0.5$ & 0.042 & 0.096 \\
\hline & $0.3,0.3,0.3,0.3,0.3$ & 0.043 & 0.092 \\
\hline & $0.1,0.3,0.5,0.3,0.1$ & 0.034 & 0.117 \\
\hline & $0.1,0.1,0.1,0.1,0.1$ & 0.008 & 0.125 \\
\hline
\end{tabular}

strata usually have power greater than that with equal allele probabilities across strata.

\section{Real example}

Ghosh reported genotype frequencies of red cell glyoxalase 1 (GLO) polymorphism from several populations. ${ }^{16} \mathrm{We}$ consider the data, reproduced in Table 5, from four populations in the Western Pacific Area. The gene frequencies of four populations highly vary from 0.0455 in the Eastern Carolines to 0.3611 in the Tokelau Islands, Samoa and Fuji in between. The estimated disequilibrium coefficients (ie, $\hat{D_{k}}$ ) in the four populations are ranging from 0.0145-0.019, which are close to zero. This seems to suggest that the homogeneity of HWE across the four populations, although the gene frequencies vary appreciably. Our proposed homogeneity score test yields $X^{2^{*}}=2.33$ with $P$-value being 0.51 . Hence, it is now safe to assume that the HWE is simultaneously valid across the four populations. We apply the Olson and Foley's test to the same glyoxalase genotype data set in the Western
Pacific Area. Function of fixation coefficients (ie, $\left(1-f_{K}\right)^{2}$ ) was adopted and the corresponding homogeneity test yields $T_{\text {homog, } a}^{2}=2.78$ with $P$-value being 0.43 . In this case, both tests reach the same conclusion.

\section{Discussion}

In practice, one is tempted to test the Hardy-Weinberg law across several independent populations without verifying the underlying assumption of homogeneity of HardyWeinberg disequilibrium across populations. Verification of the latter assumption is critical in genotype data analysis. Olson and Foley proposed a homogeneity test for this purpose. Unfortunately, our simulations show that their asymptotic version test is not reliable (ie, inflated type I error rates) even in large sample size. Although an exact version test was also proposed to overcome the liberty issue, such a test is however always conservativeness and computationally intensive for large sample sizes.

In this paper, we consider a homogeneity score test based on disequilibrium coefficients. Empirical results from our 
simulation studies support that our homogeneity score test is a reliable asymptotic testing procedure even for small sample sizes. However, our test may suffer the drawback that it may be quite conservative for rare allelic probabi-

Table 3 Empirical type I error rates for $X^{2^{*}}$ under $\mathrm{H}_{0}$

\begin{tabular}{|c|c|c|c|}
\hline$n$ & $D$ & $p$ & $\begin{array}{c}\text { Empirical } \\
\text { size }\end{array}$ \\
\hline $30,30,30$ & 0.03 & $0.5,0.5,0.5$ & 0.061 \\
\hline $50,50,50$ & & & 0.055 \\
\hline $100,100,100$ & & & 0.051 \\
\hline $30,30,30$ & -0.03 & $0.5,0.5,0.5$ & 0.048 \\
\hline $50,50,50$ & & & 0.049 \\
\hline $100,100,100$ & & & 0.050 \\
\hline $30,30,30$ & 0.03 & $0.5,0.4,0.3$ & 0.045 \\
\hline $50,50,50$ & & & 0.048 \\
\hline $100,100,100$ & & & 0.049 \\
\hline $30,30,30$ & -0.03 & $0.5,0.4,0.3$ & 0.040 \\
\hline $50,50,50$ & & & 0.042 \\
\hline $100,100,100$ & & & 0.049 \\
\hline $30,30,30,30,30$ & 0.030 & $5,0.5,0.5,0.5,0.5$ & 0.063 \\
\hline $50,50,50,50,50$ & & & 0.057 \\
\hline $100,100,100,100,100$ & & & 0.051 \\
\hline $30,30,30,30,30$ & -0.030 & $5,0.5,0.5,0.5,0.5$ & 0.049 \\
\hline $50,50,50,50,50$ & & & 0.050 \\
\hline $100,100,100,100,100$ & & & 0.050 \\
\hline $30,30,30,30,30$ & 0.030 & $5,0.4,0.3,0.4,0.5$ & 0.047 \\
\hline $50,50,50,50,50$ & & & 0.049 \\
\hline $100,100,100,100,100$ & & & 0.049 \\
\hline $30,30,30,30,30$ & -0.030 & $5,0.4,0.3,0.4,0.5$ & 0.038 \\
\hline $50,50,50,50,50$ & & & 0.042 \\
\hline $100,100,100,100,100$ & & & 0.046 \\
\hline
\end{tabular}

lities (eg, $\leqslant 0.1$ ). In this case, one may require larger sample sizes to overcome the conservativeness issue. In this regard, we also provide a sample size formula for design purpose. We have implemented the test procedures described in this manuscript in a Matlab program, which can be downloaded from the web site: http://math.nenu.edu.cn/jhguo/ program.htm.

We also applied the Kolmogorov-Smirnov test to study the asymptotic behaviors of our test (ie, $X^{2 *}$ ). Briefly, for allele frequency greater than or equal to 0.1 , we find that the asymptotic $\chi^{2}$ sampling distribution property follows for moderate sample sizes (eg, $\left.n_{k} \geqslant 50\right)$. For rare allele frequency (ie, $<0.1$ ), larger sample sizes are required. In fact, after some straightforward algebra, we observe that $H_{k D}\left(D^{*}, p_{k}{ }^{*}\right)$ has larger variance for rare $p_{k}$. This may explain the severe conservativeness of $X^{2 *}$ for rare $p_{k}$. We are now undertaking an investigation of possible modification of $X^{2 *}$ for conservative correction.

We note that exact (conditional) method works in Olson and Foley ${ }^{12}$ as they considered fixation coefficient $f$ s which in turn are odds ratio. In their case, sufficient statistics for those nuisance parameters exist and can be eliminated by conditioning on their sufficient statistics. On the contrary, we consider the disequilibrium coefficient $D$ 's, which are actually rate differences. In our case, sufficient statistics do not exist for the corresponding nuisance parameters and the exact conditional method hence is not applicable. ${ }^{17}$

Table 4 Empirical power for $X^{2 *}$

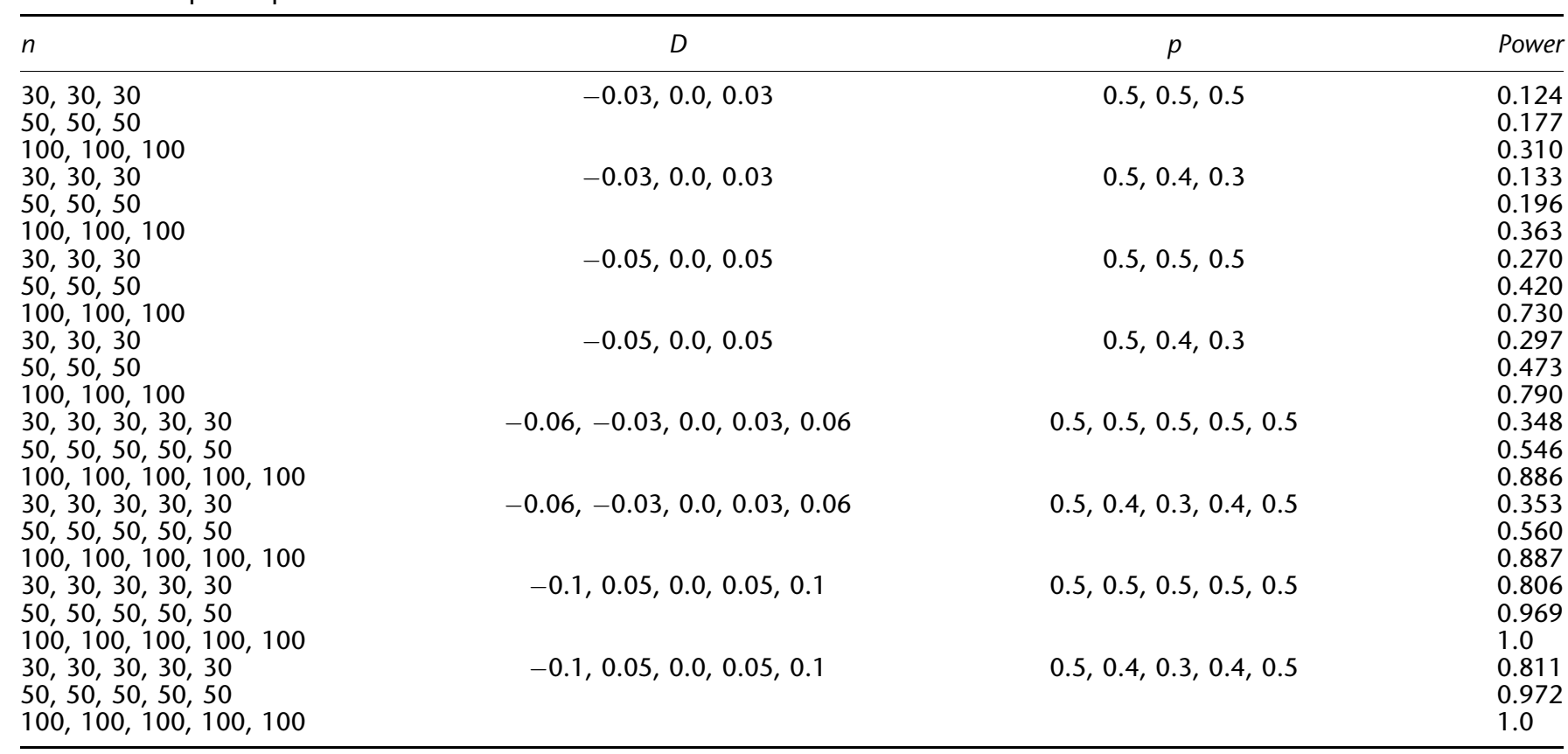


Table 5 Glyoxalase genotype data in Western Pacific Area

\begin{tabular}{lrrrrrr}
\hline Population & \multicolumn{6}{c}{ Genotype counts } \\
& $n_{k}$ & $1-1$ & $1-2$ & $2-2$ & $\hat{p}_{k}$ & \multicolumn{1}{c}{$\hat{D}_{k}$} \\
\hline Eastern Carolines & 748 & 3 & 62 & 683 & 0.0455 & 0.0019 \\
Tokelau Islands & 961 & 118 & 458 & 385 & 0.3611 & -0.0076 \\
Samoa & 101 & 4 & 39 & 58 & 0.2327 & -0.0145 \\
Fiji & 137 & 4 & 38 & 95 & 0.1679 & 0.0010 \\
\hline
\end{tabular}

Finally, the theories developed in this paper can be readily extended to genotype data with multiple alleles.

\section{Acknowledgements}

This research is supported by the NSFC (Grant Numbers 10431010, 10329102, and 10371015), the Science and Technology Keystone Fund of MOE, P.R. China (Grant Numbers 104070 and 00041), NCET-04-0310, EYTP, the Jilin Distinguished Young Scholars Program (Grant Number 20030113), and the Science Foundation for Young Teachers of NENU (Grant Number 20060101). The work of ML Tang was fully supported by a grant from the Research Grant Council of the Hong Kong Special Administration (Project Number CUHK4371/O4M) and Hong Kong Baptist University Grants FRG/ 04-05/II-01 and FRG/04-05/II-20.

\section{References}

1 Hardy GH: Mendelian proportions in a mixed population. Science 1908; 28: 49-50.

2 Weinberg W: Papers on Human Genetics. Englewood Cliffs, NJ: Prentice-Hall, 1908, In (translation by Boyer SH) On the Demonstration of Heredity in Man 1963.

3 Crow JE: Eighty years ago: the beginnings of population genetics. Genetics 1988; 119: 473-476.

4 Hernandez JL, Weir BS: A disequilibrium coefficient approach to Hardy-Weinberg testing. Biometrics 1989; 45: 53-70.

5 Olson JM: Testing the Hardy-Weinberg law across strata. Ann Hum Genet 1993; 57: 291-295.

6 Weir BS: Genetic Data Analysis II. Sunderland, MA: Sinauer Associates, Inc., 1996, pp 91-140.

7 Haldane JBS: An exact test for randomness of mating. J Genet 1954; 52: 631-635.

8 Smith CAB: A note on testing the Hardy-Weinberg law across strata. Ann Hum Genet 1970; 33: 377-383.

9 Emigh TH: A comparison of tests for Hardy-Weinberg equilibrium. Biometrics 1980; 36: 627-642.

10 Troendle JJ, Yu KF: A note on testing the Hardy-Weinberg law across strata. Ann Hum Genet 1994; 58: 397-402.

11 Nam JM: Testing a genetic equilibrium across strata. Ann Hum Genet 1997; 61: 163-170.

12 Olson JM, Foley M: Testing for homogeneity of Hardy-Weinberg disequilibrium using data sampled from several populations. Biometrics 1996; 52: 971-979.

13 Tarone RE: Homogeneity score tests with nuisance parameters. Commun Statist Theory Meth 1988; 17: 1549-1556.

14 Mantel N, Haenszel W: Statistical aspects of the analysis of data from retrospective studies of disease. J Natl Cancer Inst 1959; 22: $719-748$.

15 Guo JH, Ma YP, Shi NZ, Lau TS: Testing for homogeneity of relative difference under inverse sampling. Comput Statist Data Anal 2004; 44: 613-624.
16 Ghosh AK: Polymorphism of red cell glyoxalase 1 with special reference to South and Southeast Asian and Oceania. Hum Genet 1977; 39: 91-95.

17 Agresti A: Exact inference for categorical data: recent advances and continuing controversies. Statist Med 2001; 20: 2709-2722.

\section{Appendix A}

\section{Consistency and the condition to attain asymptotic efficiency for $D^{*}$}

Let $n_{k}=n b_{k}$, with $b_{k}>0$ and $k=1,2, \ldots, K$. The asymptotic property of $D^{*}$ is obtained under the assumption that $K$ is fixed and $n$ approaches infinity (ie, sufficiently large). Following the notation above, the Mantel-Haenszel-type estimator of $D^{*}$ can be rewritten as

$$
D^{*}=\sum_{k=1}^{K}\left(n_{k} / x_{12 k}\right)^{2} \hat{D}_{k} / \sum_{k=1}^{K}\left(n_{k} / x_{12 k}\right)^{2}
$$

By the Central Limit Theorem, $\sqrt{n}\left(y_{k}-g_{k}\right)$ has an asymptotic normal distribution, $N\left(0, \sum_{k} / b_{k}\right)$. By delta method, we obtain $\sqrt{n}\left(\hat{D}_{k}-D_{k}\right)$ has an asymptotic normal distribution with mean 0 and variance $w_{k}\left(D_{k}, p_{k}\right) / b_{k}$. As $D_{k}=D$ under $\mathrm{H}_{0}$ for $k=1,2, \ldots, K$, we can derive that $D^{*}$ is a consistent estimate of $D$. Let $w_{k}=w_{k}\left(D, p_{k}\right), v_{k}=1 /\left(p_{k} q_{k}-D\right)$. Hence, the asymptotic variance of $D^{*}$ under $\mathrm{H}_{0}$ is given by

$$
\operatorname{Asy} \operatorname{Var}\left(D^{*}\right)=\frac{\sum_{k=1}^{K} w_{k} v_{k}^{4} / b_{k}}{n\left(\sum_{k=1}^{K} v_{k}^{2}\right)^{2}}
$$

Denote the information matrix with respect to $\mathrm{D}$ and $\mathbf{p}$ under $\mathrm{H}_{0}$ by

$$
I=\left(\begin{array}{llll}
\sum_{k=1}^{K} I_{K D D} & I_{1 D p_{1}} & \ldots & I_{K D p_{K}} \\
I_{1 D p_{1}} & I_{1 p_{1} p_{1}} & \ldots & I_{K p_{1} p_{K}} \\
\vdots & \ddots & & \vdots \\
I_{K D p_{K}} & \ldots & \ldots & I_{K p_{K} p_{K}}
\end{array}\right)
$$

By inverting I, we can obtain the asymptotic variance of $\hat{D}$, which is given by

$$
\operatorname{Asy} \operatorname{Var}(\hat{D})=\frac{1}{s}\left(\sum_{k=1}^{K} b_{k} / w_{k}\right)^{-1}
$$

By the Cauchy-Schwarz inequality

$$
\left(\sum_{k=1}^{K} v_{k}^{2}\right)^{2} \leqslant\left(\sum_{k=1}^{K} w_{k} v_{k}^{4} / b_{k}\right)\left(\sum_{k=1}^{K} b_{k} / w_{k}\right)
$$

Thus, $\operatorname{Asy} \operatorname{Var}(\hat{D}) \leqslant A s y \operatorname{Var}\left(D^{\star}\right)$ and we get the sufficient and necessary condition for the asymptotic efficiency of $D^{*}$, that is, $w_{k} v_{k}^{2}=c, k=1,2, \ldots, K$, where $c$ is a constant 
independent of all the parameters. The condition is satisfied if $D=0$. From the above discussion, we know that $D^{*}$ is inefficient in general case.

\section{Appendix B}

Simple expression for $I_{k D / P_{k}}$

For the $k$ th stratum, we denote the information matrix with respect to $D_{k}$ and $p_{k}$ by

$$
I_{k}=\left(D_{k} I_{k D_{k} p_{k}} I_{k p_{k} D_{k}} I_{k p_{k} p_{k}}\right)
$$

By the property of inverse matrix, $I_{\mathrm{kD} / \mathrm{pk}}\left(D_{k}, p_{k}\right)$ is equal to the reciprocal of the $(1,1)$ element of $I_{k}^{-1}$. On the one hand, according to the property of MLEs, we have

$$
\sqrt{n_{k}}\left(\hat{D}_{k}-D_{k}, \hat{p}_{k}-p_{k}\right)^{\prime} \stackrel{D}{\longrightarrow} N\left(0, n_{k} I_{k}^{-1}\left(D_{k}, p_{k}\right)\right),
$$

where $\hat{D_{k}}=\left(4 x_{11 \mathrm{k}} x_{22 k}-x_{12 k}^{2}\right) /\left(4 \mathrm{n}_{k}^{2}\right)$ is the MLE of $D_{\mathrm{k}}$. Therefore, the asymptotic variance of $\sqrt{n_{k}} \hat{D}_{k}$ is $n_{k} / I_{k D \mid p_{k}}\left(D_{k}, p_{k}\right)$. On the other hand, let $y_{k}=\left(x_{11 k}, x_{12 k}, x_{22 k}\right) / n_{k}$, by the Central Limit Theorem, $\sqrt{n_{k}}\left(y_{k}-g_{k}\right)$ has an asymptotic normal distribution, $N\left(0, \sum_{k}\right)$, where $g_{k}=\left(p_{11 k}, p_{12 k}, p_{22 k}\right)^{\prime}$, $\sum_{k}=\operatorname{diag}\left(g_{k}\right)-g_{k} g^{\prime}{ }_{k}$. Let $c_{k}=\left.\frac{\partial D_{k}}{\partial y_{k}}\right|_{y_{k}=g_{k}}$. By delta method, we obtain $\sqrt{n_{k}}\left(\hat{D}_{k}-D_{k}\right)$ has an asymptotic normal distribution with mean 0 and variance $c^{\prime}{ }_{k} \sum_{k} c_{k}$. After simple calculation, we have $c^{\prime}{ }_{k} \sum_{k} c_{k}=w_{k}\left(D_{k}, p_{k}\right)$. Hence, we can give the exact expression $I_{k D \mid p_{k}}\left(D_{k}, p_{k}\right)=n_{k} / w_{k}\left(D_{k}, p_{k}\right)$. Naturally, the expression of $I_{k D \mid p_{k}}\left(D, p_{k}\right)$ is just $I_{k D \mid p_{k}}\left(D_{k}, p_{k}\right)$ by substituting $D$ for $D_{k}$. 